\title{
ELECTRON ACCELERATION AT CORONAL AND INTERPLANETARY SHOCK WAVES
}

\author{
G. Mann*
}

\begin{abstract}
Coronal mass ejections and/or flares are able to generate shock waves travelling through the solar corona and entering the interplanetary space in some cases. These shock waves manifest themselves in solar and interplanetary type II radio bursts. But other shock waves, e.g. planetary bow shocks and the heliospheric termination shock, are also emitting radio radiation. This property of shock waves indicates that they must be able to accelerate electrons up to suprathermal velocities. Extraterrestrial in-situ measurements of plasma waves at interplanetary shocks show that both quasi-perpendicular and quasi-parallel shocks can produce suprathermal electrons. Different acceleration mechanisms acting at quasi-perpendicular and quasiparallel shock waves are presented and, subsequently, compared in an quantitative manner.
\end{abstract}

\section{Introduction}

In the solar corona shock waves are genereated by flares and/or coronal mass ejections (CMEs). Some of them are able to penetrate into the interplanetary space, where they appear as interplanetary shocks. They manifest themselves as solar- and interplanetary type II radio bursts [Wild et al., 1959; Boischot et al., 1980a; as reviews see Bougeret, 1985; Auraß, 1992; Mann, 1995]. Figure 1 shows an example of a dynamic radio spectrum of a solar type II burst recorded by the radio spectrometer $(40-800 \mathrm{MHz})$ of the Astrophysikalisches Institut Potsdam (Germany) on July 12, 1994. The fundamental-harmonic structure of the type II burst is evidently seen. The slowly drifting band of enhanced radio emission is called the "backbone". The "herringbones" are the rapidly drifting emission stripes shooting up from the "backbone" towards higher and lower frequencies. They resemble type III radio bursts and are interpreted as highly energetic electron beams, which are accelerated at the shock wave associated with the type II burst. An example of an interplanetary type II burst recorded by the radio instrument aboard the ISEE 3 spacecraft is shown in Figure 1 of the paper by Lengyel-Frey and Stone [1985]. It appeared below

*Astrophys. Institut Potsdam, Observatorium für solare Radioastronomie, D-14473 Potsdam, FRG 


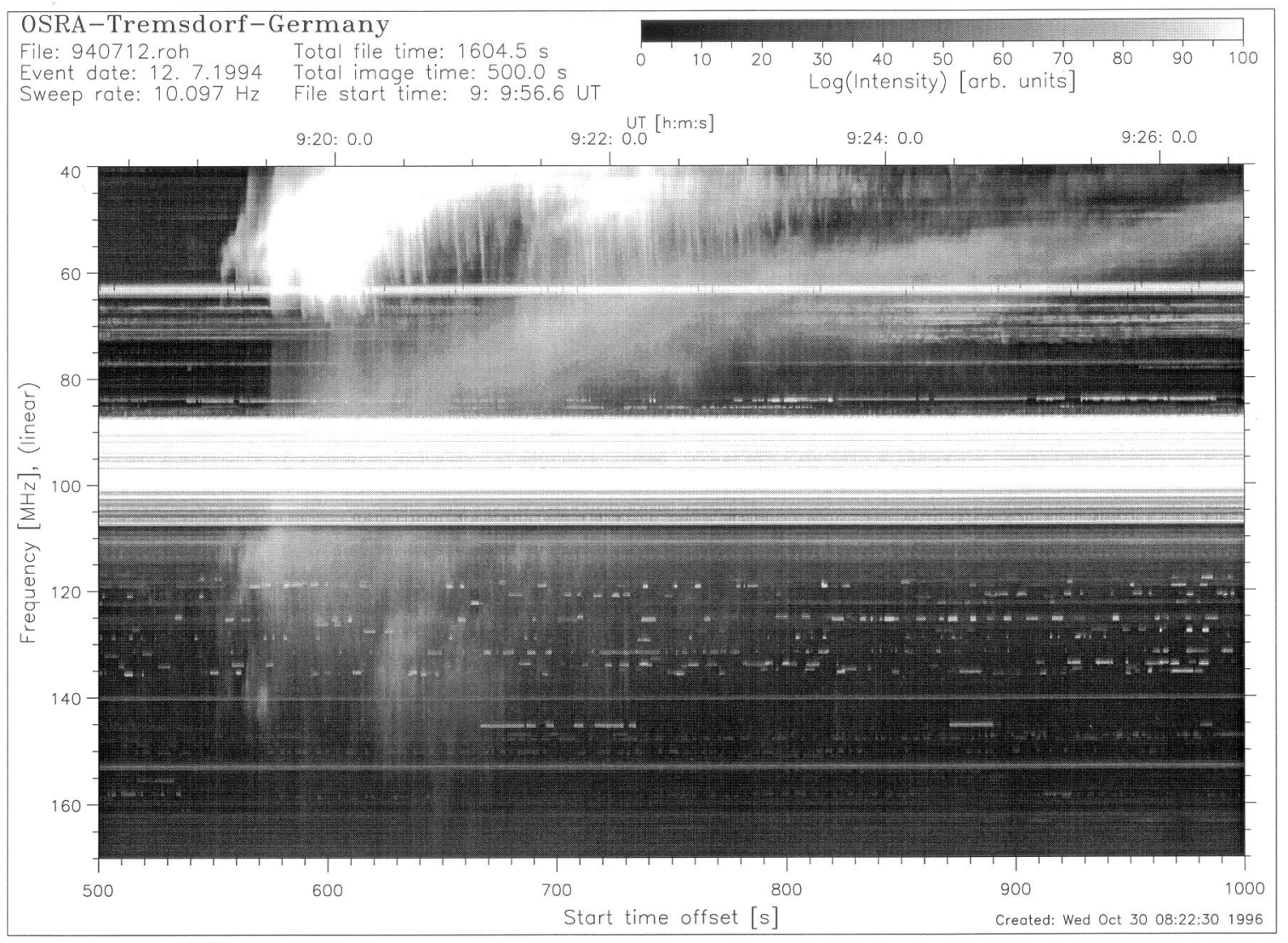

Figure 1: Dynamic radio spectrum of a solar type II radio burst recorded by the radio spectrometer of the Astrophysikalisches Institut Potsdam in the range $40-170 \mathrm{MHz}$ on July 12, 1994. The fundamental-harmonic structure of the "backbone" and the "herringbones" are evidently seen in this case.

$1 \mathrm{MHz}$. The associated emission band (i.e., "backbone") is slowly drifting from $1 \mathrm{MHz}$ towards $50 \mathrm{kHz}$ during one day.

But other shock waves are also emitting radio waves in the heliosphere, for instance, Earth's bow shock [Gurnett and Frank, 1975; Hoang et al., 1981; Treumann et al., 1986; Cairns, 1986], travelling interplanetary shocks [Cane et al., 1982], and the heliospheric termination shock [Kurth et al., 1984; Gurnett et al., 1993b].

Generally, it is assumed that the radio radiation is generated by coalescence of Langmuir waves and/or upper hybrid waves with low frequency plasma waves [Melrose, 1985]. This mechanism is responsible for the fundamental emission. On the other hand, the coalescence of two high frequency electrostatic waves leads to the harmonic radiation [Melrose, 1985]. The high frequency electrostatic plasma waves are excited by suprathermal and highly energetic electrons. Consequently, the radio radiation from shock waves in the heliosphere indicates that these shocks are able to accelerate electrons up to suprathermal velocities. 


\section{Plasma Waves at Shocks in the Heliosphere}

Plasma waves at interplanetary shocks can be studied by extra-terrestrial in-situ measurements. This was done by several spacecrafts, e.g., HELIOS 1 and 2, ISEE 3 and ULYSSES.

A very important parameter of a shock wave is the angle $\theta_{n_{s}, B_{u p}}$ between the shock normal $\vec{n}_{s}$ and the undisturbed upstream magnetic field $\vec{B}_{u p}$. Shock waves with $\theta_{n_{s}, B_{u p}}<45^{\circ}$ and $\theta_{n_{s}, B_{u p}}>45^{\circ}$ are called quasi-parallel and quasi-perpendicular, respectively. This division is not made in an arbitrary way, but determined by the ion dynamic at shock waves. Ions can be reflected by a shock wave. The guiding center motion of specularly reflected ions is directed upstream and downstream if $\theta_{n_{s}, B_{u p}}<45^{\circ}$ and $\theta_{n_{s}, B_{u p}}>45^{\circ}$, respectively [Schwartz et al., 1983].

Gurnett et al. [1979a] reported on plasma wave observations at a single interplanetary shock wave with $\theta_{n_{s}, B_{u p}}=48^{\circ}$ using data of the HELIOS mission. Furthemore, Kennel et al. [1982] investigated the plasma waves at several interplanetary shocks observed by ISEE 3 . In this sample the angles $\theta_{n_{s}, B_{u p}}$ lie in the range $22^{\circ} \leq \theta_{n_{s}, B_{u p}} \leq 88^{\circ}$. Recently, Thejappa et al. [1995] reported on plasma wave measurements at five interplanetary shocks associated with corotating interaction regions by means of the instruments aboard ULYSSES. They have angles $\theta_{n_{s}, B_{u p}}$ in the range between $19^{\circ}$ to $72^{\circ}$. In all these cases high frequency electrostatic waves (e.g., Langmuir waves and upper hybrid waves) were predominatly enhanced in the upstream region, while the low frequency plasma waves (e.g., ion acoustic waves and whistler waves) were mostly observed in the downstream region. This behaviour is evidently demonstrated in Figure 2 in the paper by Thejappa et al. [1995]. There, the electric and magnetic field spectra of a shock wave on January 22, 1993 have been presented for the upstream, transition, and downstream region. One can see that plasma waves near the electron plasma frequency are strongly enhanced in the upstream and transition region. The angle $\theta_{n_{s}, B_{u p}}$ of this shock has the value $35^{\circ}$, i.e., it is a quasi-parallel shock.

These measurements showed that high frequency electrostatic plasma waves appear in the upstream and transition region of both quasi-parallel and quasi-perpendicular shocks. Since these plasma waves near the local electron plasma frequency must be excited by suprathermal electrons, both quasi-parallel and quasi-perpendicular shock waves must be able to accelerate electrons up to suprathermal velocities.

In contradiction to these results, plasma waves at the local electron plasma frequency have only been observed near the tangential line in the upstream region of Earth's bow shock [Filbert and Kellog, 1979], i. e., in the region, where the angle $\theta_{n_{s}, B_{u p}}$ is nearly $90^{\circ}$.

\section{Shock Drift Acceleration}

A fast magnetosonic shock wave is accompanied with a compression of the density and the magnetic field. Going into the rest frame of the shock a $\vec{v}_{s} \times \vec{B}_{u p}$ electric field is appearing in the upstream region. Here, $\vec{v}_{s}$ denotes the velocity of the shock. Just this electric field is 
removed in the de Hoffmann-Teller frame. Then, a shock wave represents only a magnetic mirror in the de Hoffmann-Teller frame, i.e., it can reflect charged particles conserving the magnetic moment and the kinetic energy. Consequently, the component of the particle velocity parallel to the magnetic field changes its sign, i.e., $V_{r, \|}^{H T}=-V_{i, \|}^{H T}$, while the magnitude of the particle velocity perpendicular to the magnetic field stays unchanged, i.e., $\left(V_{r, \perp}^{H T}\right)^{2}=\left(V_{i, \perp}^{H T}\right)^{2}$, during the reflection process in the de Hoffmann-Teller frame. Finally, the transformation back into the plasma rest frame provides

$$
V_{r, \|}=2 v_{s} \cdot \sec \left(\theta_{n_{s}, B_{u p}}\right)-V_{i, \|}
$$

[Sonnerup, 1969; Holman and Pesses, 1983; Schwartz et al., 1983; Krauss-Varban, 1989]. Here, $V_{i, \|}$ and $V_{r, \|}$ denote the component of the particle velocity parallel to the upstream magnetic field before and after the reflection process, respectively. Thus, the gain of the velocity $\Delta V_{\|}=2 v_{s} \sec \left(\theta_{n_{s}, B_{u p}}\right)$ is determined by the shock speed $v_{s}$ and the angle $\theta_{n_{s}, B_{u p}}$ (cf. Eq. (1)).

The behaviour of an electron as a test particle is demonstrated in Figure 2 during a reflection at a quasi-perpendicular shock $\left(\theta_{n_{s}, B_{u p}}=85^{\circ}\right)$ with a shock speed $2.5 v_{A}\left(v_{A}\right.$, Alfvén speed). The shock is located in the $y-z$ plane. The upstream magnetic field lies in the $x-z$ plane. The initial pitch angle $\vartheta=\arctan \left(V_{i, \perp} / V_{i, \|}\right)$ of the electron is $\vartheta=45^{\circ}$. The temporal behaviour of the particle velocity parallel to the local magnetic field is shown on the right hand side. The velocity gain is determined to be $56.9 v_{A}$ and agrees well with the value resulting from Eq. (1).
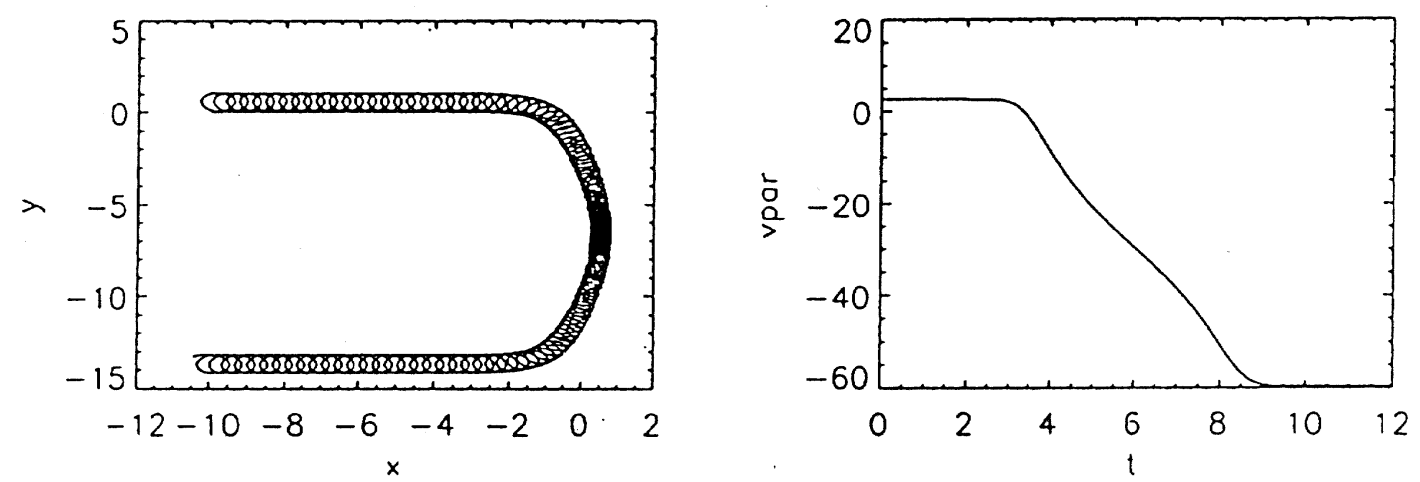

Figure 2: Trajectory of an electron in the $x$-y plane (left) during a reflection at a quasiperpendicular shock with $\theta_{n_{s}, B_{u p}}=85^{\circ}$ located in the $y-z$ plane. The upstream magnetic field lies in the $x-z$ plane. The behaviour of the particle velocity parallel to the local magnetic field during the reflection process is drawn on the right hand side. The increase of the velocity due to shock drift acceleration is evidently seen. The spatial and temporal coordinates and the velocities are normalized to the ion inertial length, the inverse proton cyclotron frequency, and the Alfvén speed $v_{A}$.

Now, the velocity gain of electrons is discussed for single reflections at coronal shock waves, travelling interplanetary shocks and Earth's bow shock, for instance.

Since solar type II radio bursts, which represent signatures of coronal shock waves, are mostly observed in the meter wave range, the plasma parameters usually found at the 
$70 \mathrm{MHz}$ level are adopted for the following estimations. Thus, a particle number density $N=6 \cdot 10^{7} \mathrm{~cm}^{-3}$, a magnetic field $B=1 \mathrm{G}$, and a temperature $T=1 \cdot 10^{6} \mathrm{~K}$ are employed as typical plasma parameters at the $70 \mathrm{MHz}$ level. They result in an Alfvén velocity $v_{A}=280 \mathrm{~km} / \mathrm{s}$, a thermal electron speed $v_{t h, e}=3900 \mathrm{~km} / \mathrm{s}$, and a plasma beta $\beta=0.5$. $v_{s}=600 \mathrm{~km} / \mathrm{s}\left(M_{A}=v_{s} / v_{A}=2.2\right)$ is a typical speed of coronal shock waves as deduced from solar type II radio bursts [Mann et al., 1994a]. Assuming for the initial electron velocity $V_{i, \|}=2^{3 / 2} \cdot v_{t h, e}=11000 \mathrm{~km} / \mathrm{s}$ one gets a velocity $V_{r, \|}=$ $14500 \mathrm{~km} / \mathrm{s}=3.7 v_{t h, e}\left(\Delta V_{\|}=3500 \mathrm{~km} / \mathrm{s}=0.9 v_{t h, e}\right)$ and $V_{r, \|}=25000 \mathrm{~km} / \mathrm{s}=6.4 v_{t h, e}$ $\left(\Delta V_{\|}=14000 \mathrm{~km} / \mathrm{s}=3.6 v_{t h, e}\right)$ after the reflection at a coronal shock wave according to Eq. (1) by using $\theta_{n_{s}, B_{u p}}=70^{\circ}$ and $\theta_{n_{s}, B_{u p}}=85^{\circ}$, respectively.

In the interplanetary medium a particle number density $N=5 \mathrm{~cm}^{-3}$, a magnetic field $B=6 \mathrm{nT}$, and a temperature $T=2 \cdot 10^{5} \mathrm{~K}$ are regarded as typical plasma parameters resulting in an Alfvén velocity $v_{A}=60 \mathrm{~km} / \mathrm{s}$, a thermal electron speed $v_{t h, e}=1800 \mathrm{~km} / \mathrm{s}$, and a plasma beta $\beta=1$. Interplanetary shock waves have typical Alfvén-Mach numbers of $M_{A}=v_{s} / v_{A}=2.4$, i.e., $v_{s}=140 \mathrm{~km} / \mathrm{s}$. If the initial velocity of the reflecting electron is $V_{i, \|}=2^{3 / 2} \cdot v_{t h, e}$ as above, i.e., $V_{i, \|}=5100 \mathrm{~km} / \mathrm{s}$, shock drift acceleration provides a final electron velocity $V_{r, \|}=5900 \mathrm{~km} / \mathrm{s}=3.2 v_{t h, e}$, i.e., $\Delta V_{\|}=800 \mathrm{~km} / \mathrm{s}=0.4 v_{t h, e}$ (cf. Eq. (1)) for $\theta_{n_{s}, B_{u p}}=70^{\circ}$. In contradiction to interplanetary shocks, Earth's bow shock is an high Alfvén-Mach number shock, i.e., $M_{A}$ is of about 7 . Then, such a shock accelerates an electron up to $V_{r, \|}=7600 \mathrm{~km} / \mathrm{s}=4.2 v_{t h, e}\left(\Delta V_{\|}=5800 \mathrm{~km} / \mathrm{s}=3.2 v_{t h, e}\right)$ by using the same values of the initial velocity and the angle $\theta_{n_{s}, B_{u p}}$, i.e., $V_{i, \|}=5100 \mathrm{~km} / \mathrm{s}$ and $\theta_{n_{s}, B_{u p}}=70^{\circ}$.

It is evidenly seen in all of these three examples that shock drift acceleration is only efficient for electrons at nearly perpendicular shocks, i.e., $\theta_{n_{s}, B_{u p}} \approx 90^{\circ}$. In other words, the velocity gain of electrons by shock drift acceleration becomes only sufficiently large at nearly perpendicular shocks under circumstances usually found in the heliosphere.

Up to now, the haviour of a single electron has been considered during the reflection at a shock wave. If a particle population with a Maxwellian distribution function

$$
f_{i}\left(V_{i, \|}, V_{i, \perp}\right)=\frac{1}{\left(2 \pi v_{t h, e}^{2}\right)^{3 / 2}} \cdot \exp \left\{\frac{-\left(V_{i, \|}^{2}+V_{i, \perp}^{2}\right)}{2 v_{t h, e}^{2}}\right\}
$$

( $v_{t h, e}$, thermal speed of electrons) is existing as the initial state in the upstream region, a shifted loss-cone distribution

$$
\begin{array}{r}
f_{r}\left(V_{r, \|}, V_{r, \perp}\right)=\frac{\Theta\left(V_{r, \|}-U_{s}\right)}{\left(2 v_{t h, e}^{2}\right)^{3 / 2}} \cdot \Theta\left(V_{r, \perp}-\left[V_{r, \perp}-U_{s}\right] \cdot \tan \alpha_{l c}\right) \\
\times \exp \left\{\frac{-\left[\left(-V_{r, \|}+2 U_{s}\right)^{2}+V_{r, \perp}^{2}\right]}{2 v_{t h, e}^{2}}\right\}
\end{array}
$$

$\left(U_{s}=v_{s} \sec \left(\theta_{n_{s}, B_{u p}}\right)\right)$ results for the reflected particles due to shock drift acceleration in the upstream region [Leroy and Mangeney, 1984; Wu, 1984]. Here, $\Theta$ denotes the well-known step-function. $V_{r, \|}$ and $V_{r, \perp}$ are the velocity components parallel and perpendicular with respect to the upstream magnetic field for the reflected electrons, respectively. The shape of the shifted loss-cone distribution is illustrated in Figure 3. As already mentioned the 

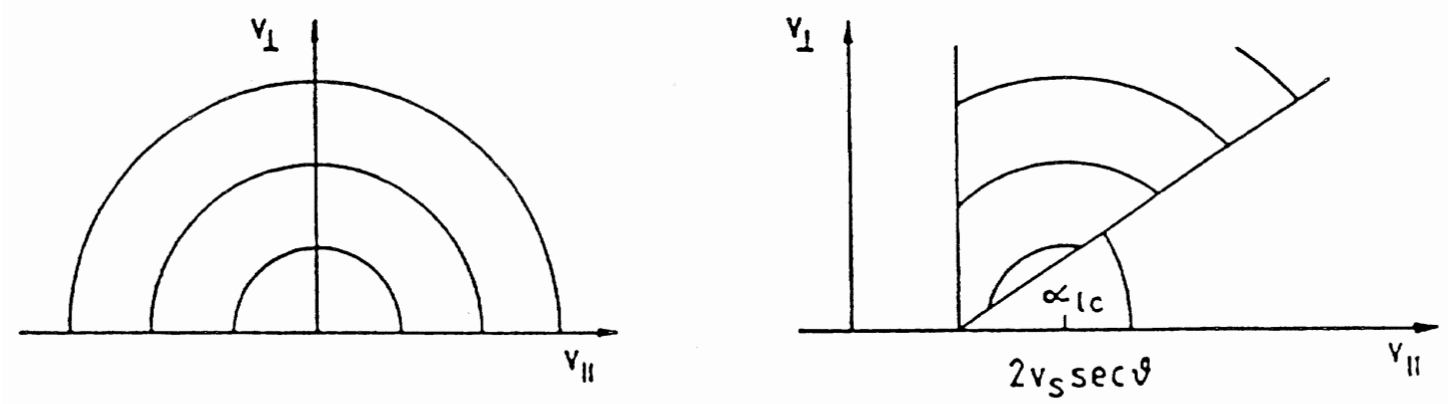

Figure 3: Illustration of a shifted loss-cone distribution (right) (cf. Eq. (3)) resulting from a Maxwellian distribution (left) (cf. Eq. (2)) by shock drift acceleration. $v_{\|}$and $v_{\perp}$ denote the velocity component parallel and perpendicular to the magnetic field, respectively.

fast magnetosonic shock represents a magnetic mirror in the de Hoffmann-Teller frame. Consequently, particles with a pitch angle $\vartheta=\arctan \left(V_{i, \perp}^{H T} / V_{i, \|}^{H T}\right)>\alpha_{l c}$ are only reflected by the shock. The second $\Theta$-function in Eq. (3) takes into account, that electrons fulfilling the above condition for their pitch angle $\vartheta$ are only reflected and, subsequently, appear as accelerated particles in the upstream region. The other ones penetrate into the downstream region. Here, the loss-cone angle $\alpha_{l c}$ is defined by the jump of the magnetic field across the shock according to $\alpha_{l c}=\arcsin \left[\left(B_{\text {up }} / B_{\text {down }}\right)^{1 / 2}\right]\left(B_{u p}\right.$ and $B_{\text {down }}$ represent the magnitude of the undisturbed magnetic field in the up- and downstream region, respectively.) Such a shifted loss-cone distribution is unstable and able to excite upper hybrid waves [Benz and Thejappa, 1988]. The appearance of a shifted loss-cone distribution near the tangential line $\left(\theta_{n_{s}, B_{u p}} \approx 90^{\circ}\right)$ upstream of Earth's bow shock has been confirmed by in-situ measurements aboard the WIND spacecraft [Larson et al., 1996].

\section{Electron Acceleration at Quasi-Parallel Shocks}

\subsection{Structure of Quasi-Parallel Shock Waves}

Interplanetary shocks and planetary bow shocks can be investigated by extra-terrestrial in-situ measurements as special examples of collisionless shocks in space plasmas (cf. Kennel et al. [1985] as a review). Earth's bow shock is the mostly observed shock in the heliosphere. Because of its curvature it has regions of a quasi-parallel $\left(\theta_{n_{s}, B_{u p}}<45^{\circ}\right)$ and quasi-perpendicular $\left(\theta_{n_{s}, B_{u p}}>45^{\circ}\right)$ shock geometry.

In Figure 4 the behaviour of the magnitude of the magnetic field is presented during a quasi-parallel crossing of Earth's bow shock. The data were recorded by the AMPTE/IRM satellite. The arrow indicates the shock transition as deduced from the plasma data. One can see, that a quasi-parallel, collisionless shock is accompanied by a strong variation of the magnetic field (strong magnetohydrodynamic turbulence) in the up- and downstream region. Recently, so-called SLAMS (an abbreviation of Short Large Amplitude Magnetic Field Structures) have been observed as a common feature in the vicinity of the quasi-parallel region of Earth's bow shock [Schwartz et al., 1992]. For example, SLAMS 


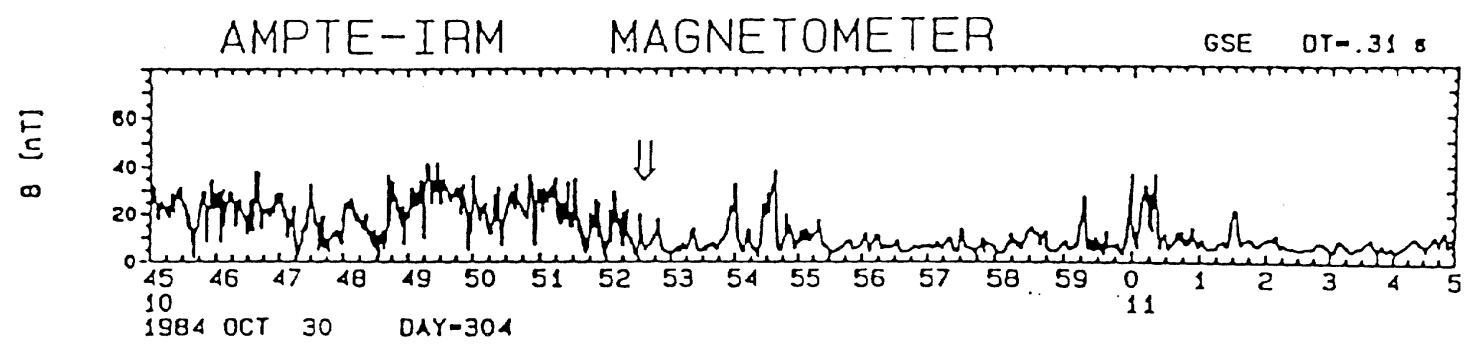

Figure 4: Behaviour of the magnitude of the magnetic field during a crossing of the quasi-parallel Earth's bow shock as recorded by the magnetometer aboard the AMPTE/IRM satellite. The arrow is indicating the shock transition as deduced from the plasma data.

appeared at 10:54:50, 10:59:15, and 11:01:30 UT on October 30, 1984 (cf. Figure 4). Schwartz and Burgess [1991] argued, that a quasi-parallel shock should be regarded as a patchwork of SLAMS. SLAMS represent strong magnetic field compressions of typically $3 \times B_{u p}$ during a time of about $10 \mathrm{~s}$, i. e., their spatial width is 10 ion inertial length (1 ion inertial length $=1 \cdot c / \omega_{p i} ; c$, velocity of light; $\omega_{p i}$, proton plasma frequency) [Schwartz et al., 1992; Mann et al., 1994b]. Note that their magnetic field compression is greater than the jump of the magnetic field according to the Rankine-Hugoniot relationship, i. e., $B_{\max } / B_{\text {up }}>\left(B_{\text {down }} / B_{\text {up }}\right)_{R H}$. Here, $B_{\max }$ and $B_{\text {down }}$ are the maximum of the magnetic field magnitude within an individual SLAMS and the mean value of the magnetic field magnitude in the downstream region, respectively. SLAMS are propagating quasi-parallel to the undisturbed upstream magnetic field with a typical velocity of 3 times the Alfvén speed in the plasma rest frame. Note, that their velocity is a monotonically increasing function of the magnetic field compression $B_{\max } / B_{\text {up }}$ [Schwartz et al., 1992; Mann et al., 1994b]. Thus, SLAMS represent moving magnetic mirrors, at which particles can be reflected and accelerated.

\subsection{Mirror Acceleration}

As mentioned above, two neighbouring SLAMS with different magnetic field compressions would have a non-vanishing relative velocity and, consequently, represent converging magnetic mirrors, which are able to accelerate charged particles as originally proposed by Fermi [1949].

Such a system of two neighbouring SLAMS approaching to each other with a velocity $\Delta V_{S}$ is illustrated in Figure 5. The SLAMS are initially separated by a distance $L_{0}$. In the plasma rest frame the SLAMS "S1" is propagating faster than the SLAMS "S2" because of its greater magnetic field compression as shown in Figure 5. Now, the multiple reflection of an electron between these two SLAMS is considered. The electron starts with the velocity $V_{0}$ at the SLAMS "S1" and is moving towards the SLAMS "S2", which is met at a distance $L^{*}$. Then, it is reflected at the SLAMS "S2" and receives a velocity gain due to shock drift acceleration. Subsequently, it is moving with a higher velocity back towards the SLAMS "S1", at which it is reflected again. Thus, the velocity gain is $\Delta V=2\left[V_{S 1} \sec \left(\theta_{1}\right)-V_{S 2} \sec \left(\theta_{2}\right)\right]$ per revolution. Here, $V_{S 1}, \theta_{1}, V_{S 2}$, and $\theta_{2}$ denote the 


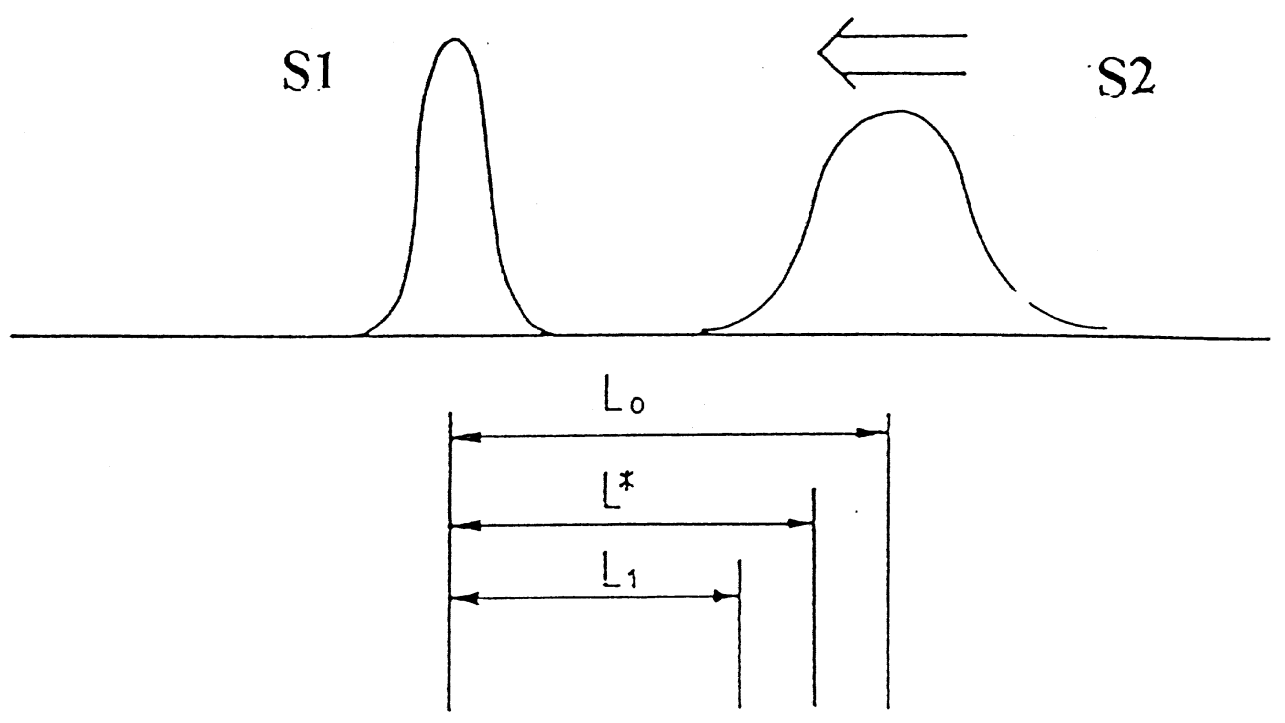

Figure 5: Illustration of two approaching SLAMS "S1" and "S2" with a relative velocity $\Delta V_{S}$.

velocity and the angle between the propagation direction and the unperturbed magnetic field of the SLAMS "S1" and "S2", respectively. After the first revolution the distance between these two neighbouring SLAMS is diminished up to $L_{1}$, which is determined by

$$
L_{1}=L_{0} \cdot\left[1-\frac{\Delta V_{S}}{\left(\Delta V_{S}+V_{0}\right)} \cdot\left\{\frac{2 V_{0}+\Delta V}{v_{0}+\Delta V}\right\}\right]
$$

The first revolution lasts $t_{1}=\left(L_{0}-L_{1}\right) / \Delta V_{S}$ with $\Delta V_{S}=V_{S 1}-V_{S 2}$. During the $(\mathrm{N}+1)$ th revolution, which lasts $t_{N+1}=\left(L_{0}-L_{N+1}\right) / \Delta V_{S}$, the particle receives a velocity gain $V_{N+1}=V_{N}+\Delta V$ and the distance between the two neighbouring SLAMS is diminished to

$$
L_{N+1}=L_{N} \cdot\left[1-\frac{\Delta V_{S}}{\Delta V_{S}+V_{N}} \cdot\left\{\frac{V_{N}+V_{N+1}}{V_{N+1}}\right\}\right]
$$

[Mann and Claßen, 1995]. The process will be finished if the distance between SLAMS $L_{N+1}$ is reaching a minimum value $L_{E}$, i. e., if $L_{N+1} \leq L_{E}$. The numerical solution of the iteration defined by Eq. (5) is depicted for two different initial electron velocities $V_{0}=26 v_{A}$ and $V_{0}=52 v_{A}\left(v_{A}\right.$, Alfvén speed in the upstream region) in Figure 6 . It is evidently seen that the particle is non-uniformly accelerated by the multiple encounters between these two SLAMS. This mechanism can only act at quasi-parallel collisionless shocks, because a strong MHD turbulence including SLAMS is only appearing at such kind of shocks.

\subsection{Discussion}

The acceleration mechanism presented in the previous Subsection will be discussed for coronal and interplanetary shocks as well as for Earth's bow shock, where the same plasma parameters are used as introduced in Section 3. 


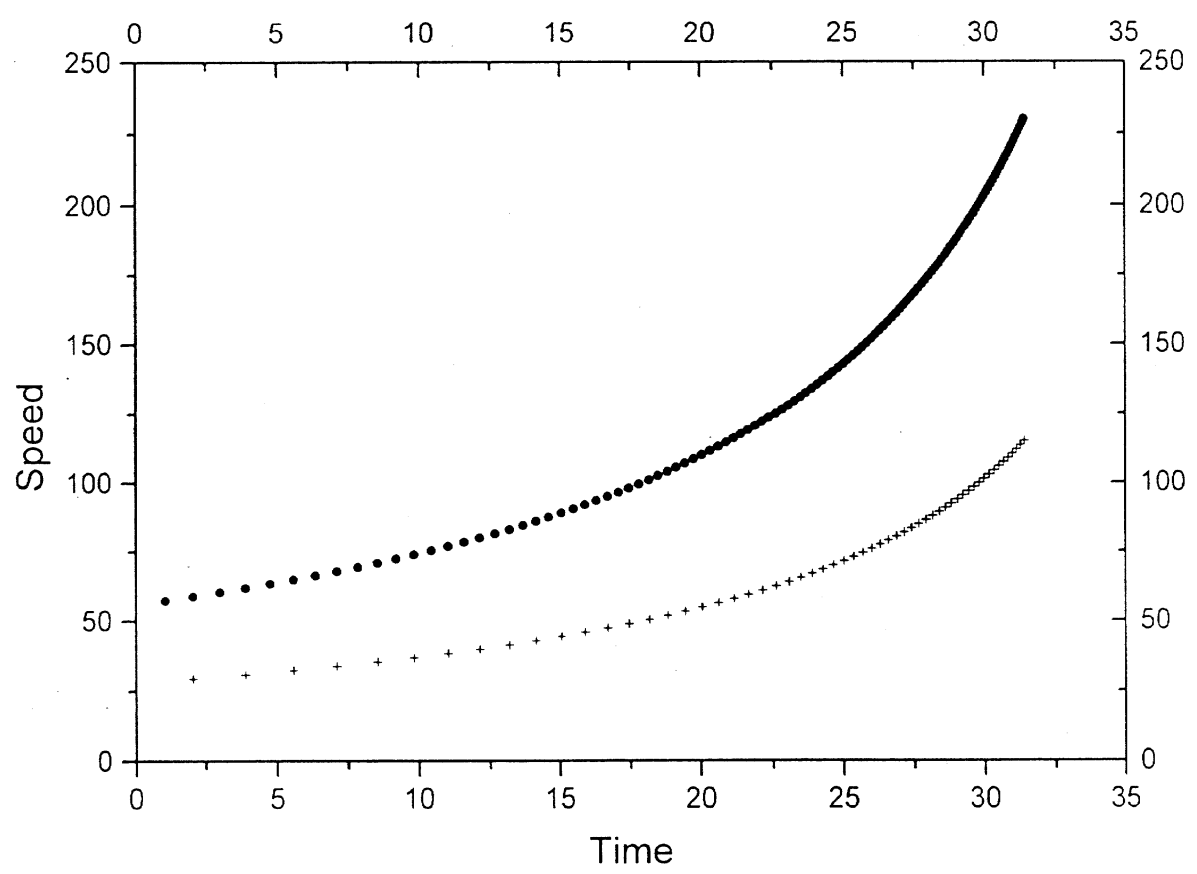

Figure 6: Velocity-time diagram as resulting from Eq. (5) with two different initial velocities $V_{0}=28(+)$ and $V_{0}=56(\bullet)$. Velocity and time are normalized with respect to the upstream Alfvén speed and the inverse proton cyclotron frequency, respectively.

In the solar corona, the plasma parameters mentioned in Section 3 provide for the ion inertial length $d_{i}=3000 \mathrm{~cm}$, the electron gyroradius $r_{L, e}=v_{t h, e} / \omega_{c i}=25 \mathrm{~cm}$, and the proton cyclotron frequency $\omega_{c i}=9.6 \cdot 10^{3} \mathrm{~s}^{-1}$. Since SLAMS have a typical width of 10 ion inertial lengths [Mann et al., 1994b], the condition of conservation of the magnetic moment, i. e., $r_{L, e}=|\nabla \vec{B}| /|\vec{B}| \ll 1$ or $r_{L, e} \ll 10 d_{i}$ is well fulfilled for SLAMS under coronal circumstances. Numerical particle simulations by Scholer et al. [1992] and Scholer [1993] showed, that SLAMS are growing from ULF upstream waves. According to these simulations, the initial distance of two neighbouring SLAMS is roughly $30 d_{i}$, and they are approaching together up to a distance of $8 d_{i}$ during their movement towards the shock front, i. e., $L_{0}=30 d_{i}$ and $L_{E}=8 d_{i}$, and, subsequently, entering into the downstream region. According to the results of the numerical particle simulations [Scholer et al., 1992; Scholer, 1993] a relative velocity $\Delta V_{S}=0.7 v_{A}$ between two SLAMS and an angle $\theta_{1} \approx \theta_{2}=$ $20^{\circ}$, and, consequently, $\Delta V=1.5 v_{A}$ seem to be appropriate for the parameters of SLAMS. Then, an electron is accelerated from an initial velocity $V_{0}=2^{3 / 2} v_{t h, e}=11000 \mathrm{~km} / \mathrm{s}$ up to $V_{E}=45400 \mathrm{~km} / \mathrm{s}=11.7 v_{t h, e}=0.15 \mathrm{c}$ within a time of $3.3 \cdot 10^{-3} \mathrm{~s}$ by this mirror mechanism [Mann and Claßen, 1995]. Thus, the velocity gain is $34370 \mathrm{~km} / \mathrm{s}=8.8 v_{t h, e}$. The final velocity corresponds to a kinetic energy of $6 \mathrm{keV}$. The "herringbones" seen at solar type II radio bursts are regarded as electrons accelerated at a shock wave in the solar corona. Cairns and Robinson [1987] deduced that electrons with velocities of about $0.2 c$ are responsible for the "herringbones". Therefore, Mann and Claßen [1995] argued that the acceleration mechanism presented in Subsection 4.2 is able to produce the highly energetic electrons needed for the "herringbones" in solar type II radio bursts. 
The plasma parameters usually found in the interplanetary space (cf. Section 3) provide an ion inertial length of $d_{i}=100 \mathrm{~km}$, an electron gyroradius $r_{L, e}=1.7 \mathrm{~km}$, and a proton cyclotron frequency $\omega_{c i}=0.57 \mathrm{~s}^{-1}$. Employing the same parameters of the SLAMS as done in the previous paragraph, the mirroring of electrons between two neighbouring SLAMS accelerate an electron with the initial velocity $V_{0}=2^{3 / 2} \cdot v_{t h, e}=5100 \mathrm{~km} / \mathrm{s}$ up to a final velocity $V_{E}=21000 \mathrm{~km} / \mathrm{s}$ during a time of $58 \mathrm{~s}$. This corresponds to a kinetic energy of $1,2 \mathrm{keV}$. The velocity gain is found to be $15900 \mathrm{~km} / \mathrm{s}=8.8 v_{t h, e}$.

In order to discuss the results of the electron acceleration mechanism (cf. Subsection 4.2) in the case of Earth's bow shock, the plasma parameters introduced for the interplanetary space are used, but the parameters of the SLAMS must be changed, since it is a high Mach number shock. Thus, $\Delta V_{S}=3.0 v_{A}$ is chosen for the relative velocity between two neighbouring SLAMS. Then, the velocity gain per revolution is $\Delta V=6.4 v_{A}$ by using $\theta_{1} \approx \theta_{2}=20^{\circ}$ Thus, an electron with an initial velocity $V_{0}=5100 \mathrm{~km} / \mathrm{s}$ is accelerated up to a final velocity $V_{E}=21400 \mathrm{~km} / \mathrm{s}$ during a time of $12.8 \mathrm{~s}$. This corresponds to a kinetic energy of $1.3 \mathrm{keV}$. The velocity gain is $16300 \mathrm{~km} / \mathrm{s}=9.1 v_{t h, e}$.

Up to now the behaviour of a single electron has been considered under circumstances of different shock waves in the heliosphere. What kind of distribution function is established by the accelerated electrons in the upstream region in the case of the mechanism presented in Subsection 4.2? A Maxwellian distribution (cf. Eq. (2)) is assumed to be initially between the two neighbouring SLAMS. Basically, the velocity gain is generated by shock drift acceleration. The total velocity gain is caused by accumulating energy due to the multiple encounters (mirroring) with these SLAMS. Consequently, only the component of the particle velocity parallel to the magnetic field is increasing. This leads to a continuous decrease of the pitch angle $\vartheta=\arctan \left(V_{i, \perp} / V_{i, \|}\right)$. If the actual pitch angle $\vartheta$ becomes smaller than the loss-cone angle $\alpha_{l c, S L A M S}$, the particle leaves the acceleration region between the two neighbouring SLAMS and can subsequently penetrate into the upstream region. The loss-cone angle $\alpha_{l c, S L A M S}$ is defined by the magnetic field compression within the SLAMS, i. e., $\alpha_{l c, S L A M S}=\arcsin \left[\left(B_{u p} / B_{\text {max }}\right)^{1 / 2}\right]$. Thus, the particles leaving the acceleration region have a pitch angle $\vartheta \approx \alpha_{l c, S L A M S}$, i. e., all accelerated electrons are located on a cone mantle in the velocity space. This process is illustrated in Figure 7 . The acceleration mechanism proposed in Subsection 4.2 is producing a so-called cone mantle shaped distribution

$$
f_{r}=\frac{\Theta\left(V_{r, \|}\right)}{\left(2 \pi v_{t h, e}^{2}\right)^{3 / 2}} \cdot \delta\left(V_{r, \perp}-V_{r, \|} \tan \alpha_{l c, S L A M S}\right) \cdot \exp \left\{\frac{-\left[\left(V_{r, \|} / \nu\right)^{2}+V_{r, \perp}^{2}\right]}{2 v_{t h, e}^{2}}\right\}
$$

for the accelerated electrons [Mann and Claßen, 1995]. $\delta$ denotes the well-known Dirac delta function. Here, $\nu$ is a factor determined by the ratio $L_{0} / L_{E}$. It has a typical value of 4. The distribution function (cf. Eq. (6)) has a beam like (i. e., $\partial f_{r} / \partial V_{r, \|}>0$ ) and a loss-cone like (i. e., $\left.\partial f_{r} / \partial V_{r, \perp}>0\right)$ part. Therefore, it is unstable and able to excite both upper hybrid and Langmuir waves [Marsch, 1990]. Thus, the cone mantle shaped distribution is different from a pure beam and loss-cone distribution. 


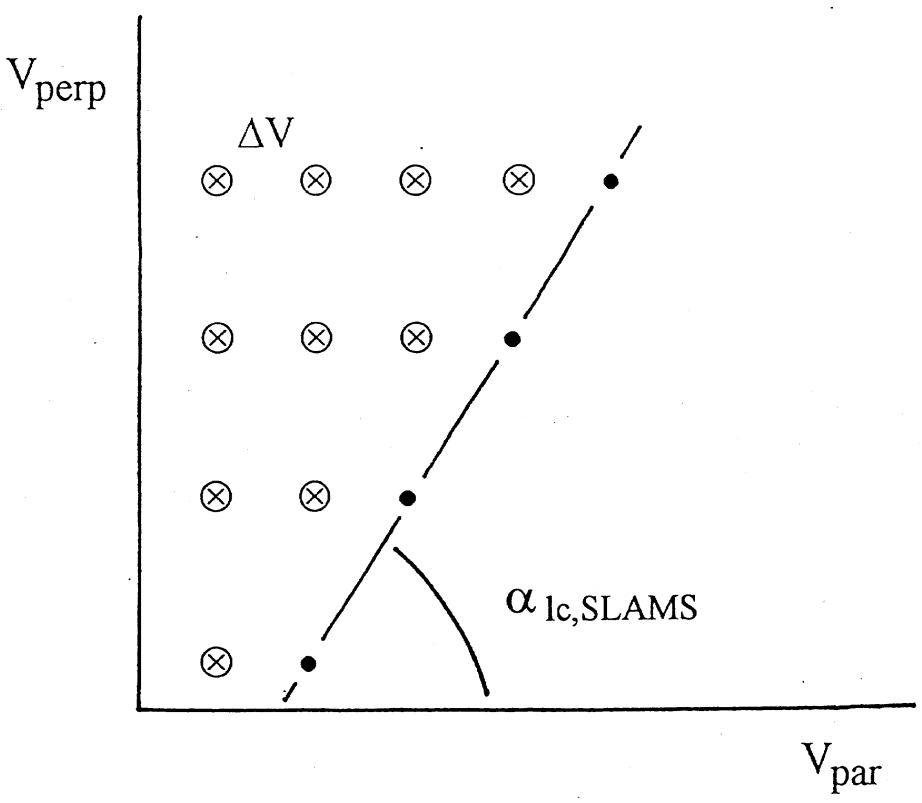

Figure 7: Illustration of the mirror acceleration in the velocity space, i. e., $V_{\perp}-V_{\|}$space. The signs " $\otimes$ " are indicating the location of individual particles in the velocity space during the mirroring. After each revolution the particle receives a gain $\Delta V$ of the velocity component parallel to the magnetic field as illustrated by the $\otimes$ signs. If the pitch angle $\vartheta=\arctan \left(V_{\perp} / V_{\|}\right)$ becomes smaller than the loss-cone angle $\alpha_{l c, S L A M S}$ they leave the acceleration region. Thus, the accelerated particles are located around a cone mantle as designed by " $\bullet$ 's".

\section{Final Discussion}

As mentioned in the Introduction and, especially, in Section 2 high frequency electrostatic waves have been observed by extra-terrestrial in-situ measurements upstream of interplanetary shocks and Earth's bow shock. Since these waves can only be excited by suprathermal electrons, these plasma wave measurements showed that both quasiperpendicualr and quasi-parallel shocks accelerate electrons up to suprathermal velocities. Furthermore, the "herringbones" appearing at solar type II radio bursts represent signatures of electron beams which are produced by the coronal shock wave associated with the type II burst. Two special acceleration mechanisms (shock drift acceleration and mirror acceleration) acting at quasi-perpendicular and quasi-parallel, collisionless shocks in space plasmas are presented in Section 3 and Subsection 4.2, respectively. Their relevance for electron acceleration has been discussed for three examples, i. e., coronal shock waves, interplanetary shocks and Earth's bow shock. In all of these cases it has been shown that shock drift acceleration is only efficient at nearly perpendicular shocks, i. e., at shocks with an angle $\theta_{n_{s}, B_{u p}} \approx 90^{\circ}$. Shock drift acceleration provides only a velocity gain of about $1 \cdot v_{t h, e}\left(v_{t h, e}\right.$, thermal electron velocity) for quasi-perpendicluar shocks with $\theta_{n_{s}, B_{u p}}<80^{\circ}$. These estimations demonstrate, that shock drift acceleration cannot produce the suprathermal elctrons, which are needed for the generation of the high frequency plasma waves observed at shock waves in the heliosphere. On the other hand, the mirror acceleration acting only at quasi-parallel shocks (with $\theta_{n_{s}, B_{u p}}<45^{\circ}$ ) provides a velocity 
gain of about $10 v_{t h, e}$ in all of the examples discussed. Thus, these velocities of the accelerated electrons are suprathermal enough, in order to excite the high frequency plasma waves. According to this argument, the mirror acceleration seems to be appropriate for producing the suprathermal electrons, which excite the high frequency plasma waves observed at different kinds of shock waves in the heliosphere. But, this mechanism can only act at quasi-parallel shocks. Since the high frequency plasma waves are also appearing at shocks with $45^{\circ}<\theta_{n_{s}, B_{u p}}<80^{\circ}$, there must be another acceleration mechanism predominatly acting at quasi-perpendicular shock waves. But such a mechanism is unknown up to now.

Acknowledgements: The author would like to express his thanks to Dr. H.-T. Claßen and P. Hackenberg for preparing the Figure 1 and for the careful reading of the manuscript. 